\title{
Realization Spaces of Arrangements of Convex Bodies*
}

\author{
Michael Gene Dobbins ${ }^{1}$, Andreas Holmsen ${ }^{2}$, and Alfredo Hubard ${ }^{3}$ \\ 1 GAIA, POSTECH \\ South Korea \\ dobbins@postech.ac.kr \\ 2 Department of Mathematical Sciences, KAIST \\ South Korea \\ andreash@kaist.edu \\ 3 GEOMETRICA, INRIA Sophia-Antipolis \\ France \\ alfredo.hubard@inria.fr
}

\begin{abstract}
We introduce combinatorial types of arrangements of convex bodies, extending order types of point sets to arrangements of convex bodies, and study their realization spaces. Our main results witness a trade-off between the combinatorial complexity of the bodies and the topological complexity of their realization space. On one hand, we show that every combinatorial type can be realized by an arrangement of convex bodies and (under mild assumptions) its realization space is contractible. On the other hand, we prove a universality theorem that says that the restriction of the realization space to arrangements of convex polygons with a bounded number of vertices can have the homotopy type of any primary semialgebraic set.
\end{abstract}

1998 ACM Subject Classification G.2.1 Combinatorics, F.2.2 Nonnumerical Algorithms and Problems

Keywords and phrases Oriented matroids, Convex sets, Realization spaces, Mnev's universality theorem

Digital Object Identifier 10.4230/LIPIcs.SOCG.2015.599

\section{Introduction}

We introduce a generalization of order types that provides a framework to study arrangements of convex sets and their convex dependencies. The notion we introduce is closely related to wiring diagrams [7] or primitive sorting networks [18]. It is also related to double pseudoline arrangements introduced by Pocchiola and Habert [14] and double allowable sequences introduced by Goodman and Pollack [11]. These related notions have applications in the study of visibility, transversal, and separation properties of convex sets $[2,23,22,16]$. The generalization of order type studied here was fundamental to the authors' work on

\footnotetext{
* M. G. Dobbins was supported by NRF grant 2011-0030044 (SRC-GAIA) funded by the government of South Korea. A. Holmsen was supported by Basic Science Research Program through the National Research Foundation of Korea funded by the Ministry of Education, Science and Technology (NRF2010-0021048). A. Hubard was supported by Fondation Sciences Mathématiques de Paris and by the Advanced Grant of the European Research Council GUDHI (Geometric Understanding in Higher Dimensions).
} 
generalizations of the Erdős-Szekeres theorem to arrangements of convex sets in the plane $[4,3]$. In this paper, we address the relevant realizability questions. ${ }^{1}$

Two indexed point sets $P=\left\{p_{1}, p_{2} \ldots p_{n}\right\}$ and $Q=\left\{q_{1}, q_{2} \ldots q_{n}\right\}$ in the plane are said to have the same order type when for every triple $(i, j, k)$ the orientation of the triples $p_{i}, p_{j}, p_{k}$ and $q_{i}, q_{j}, q_{k}$ coincides. Equivalently by projective duality, a point set $P$ corresponds to a dual family $P^{*}$ of oriented great circles in the sphere, and point sets $P$ and $Q$ have the same order type when the families $P^{*}$ and $Q^{*}$ subdivide the sphere in the same way. That is, when there is a self-homeomorphism of the sphere that sends each cell of $P^{*}$ to a corresponding cell of $Q^{*}$ and preserves orientations. A number of geometric properties of point sets which are important in combinatorial convexity, incidence geometry and algorithms depend solely on the order type of the point sets and not on actual coordinates of the points.

More generally, we say that a sign function $\chi: \mathfrak{L}^{3} \rightarrow\{+, 0,-\}$ is an order type when it satisfies the axioms of rank 3 acyclic chirotopes [1, page 126] [18, Chapter 10]. Specifically, $\chi$ is alternating, satisfies the Grassman-Plücker Relations, is acyclic (a restatement of Radon's partition theorem in terms of orientations), and is not identically zero. Order types that satisfy $\chi(i, j, k) \neq 0$ for any $i, j, k$ distinct are called simple, and are equivalent to Donald Knuth's CC-systems [18].

Like simple order types, combinatorial types are finite combinatorial objects that can be associated to families of geometric objects, namely arrangements of convex bodies, which are assumed to satisfy certain genericity conditions. These will be defined precisely in Section 2, but for now we describe the equivalence relation that combinatorial types induce on arrangements of convex bodies. To do so, we define a duality for convex bodies that is analogous to projective duality for points in the plane. The dual support curve $A^{*}$ of a convex body $A$ in the plane, is the graph of its support function $h_{A}: \mathbb{S}^{1} \rightarrow \mathbb{R}^{1}$, $h_{A}(\theta):=\max _{p \in A}\langle\theta, p\rangle$ on the cylinder $\mathbb{S}^{1} \times \mathbb{R}^{1}$, where $\mathbb{S}^{1}$ is the unit circle and $\langle\cdot, \cdot\rangle$ is the standard inner product. In this way, every arrangement $\mathcal{A}=\left\{A_{1}, \ldots, A_{n}\right\}$ corresponds to the dual support system $\mathcal{A}^{*}=\left\{A_{1}^{*}, \ldots, A_{n}^{*}\right\}$ of curves on the cylinder $\mathbb{S}^{1} \times \mathbb{R}^{1}$ given by the graphs of the functions $\left\{h_{A_{1}}, h_{A_{2}}, \ldots h_{A_{n}}\right\}$. In the other direction, not all functions $h: \mathbb{S}^{1} \rightarrow \mathbb{R}^{1}$ are support functions, but we have the following sufficient conditions.

- Remark. Blashke showed that if $h: \mathbb{S}^{1} \rightarrow \mathbb{R}^{1}$ is $C^{2}$-smooth and $h+h^{\prime \prime}>0$, then $h$ is the support function of a planar curve with curvature bounded by $\frac{1}{h+h^{\prime \prime}}$ [12, Lemma 2.2.3]. Hence, by adding a sufficiently large constant to a family of smooth functions, we can ensure the family is the dual support system of an arrangement of convex bodies.

The combinatorial type of an arrangement of bodies $\operatorname{ct}(\mathcal{A})$ is, essentially, a combinatorial encoding of the subdivision of the cylinder $\mathbb{S}^{1} \times \mathbb{R}^{1}$ by the dual support curves $\mathcal{A}^{*}$. For now, we take the following theorem as an alternative topological definition.

- Theorem 1. Two arrangements of convex bodies $\mathcal{A}$ and $\mathcal{B}$ have the same combinatorial type if and only if their dual systems $\mathcal{A}^{*}$ and $\mathcal{B}^{*}$ are related by a self-homeomorphism of the cylinder that preserves orientation and $+\infty$.

Here, we say that a self-homeomorphism $\phi: \mathbb{S}^{1} \times \mathbb{R}^{1} \rightarrow \mathbb{S}^{1} \times \mathbb{R}^{1}$ preserves $+\infty$ when for $y$ sufficiently large the second coordinate of $\phi(\theta, y)$ is positive for all $\theta$. Equivalently, $\phi$ preserves the counter-clockwise orientations of the support curves.

In the case of points, the duality that we defined through support functions is the usual projective duality renormalized to be on the cylinder. Consequently, two generic point sets

1 The full details of the proofs of our results will appear in the journal version. 


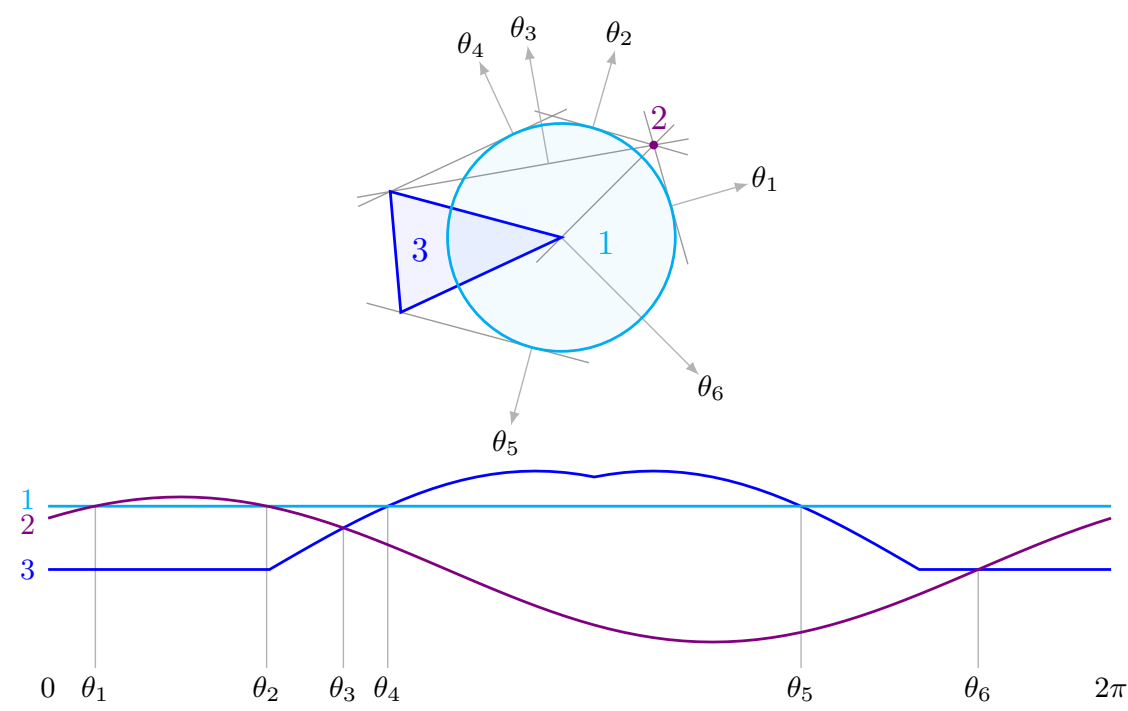

Figure 1 Top: An arrangement $\mathcal{A}$ and its common supporting tangents. Bottom: Its dual support system $\mathcal{A}^{*}$.

have the same order type if and only if they have the same combinatorial type. Specifically, a point in the plane can be represented in homogeneous coordinates by a line in $\mathbb{R}^{3}$, and its dual support curve is the intersection of the orthogonal complement of this line with the cylinder embedded in $\mathbb{R}^{3}$. The same relationship holds between a body in the plane represented by a cone in $\mathbb{R}^{3}$ and the body's dual support curve represented by its polar cone.

Although combinatorial types of arrangements are more general objects than simple order types, we associate an order type to the following class of arrangements. We say a triple of bodies is orientable when it has the combinatorial type of three generic points, and we say an arrangement is orientable when it consists of at least three bodies and every triple is orientable. In this case, every triple $\left\{A_{i}, A_{j}, A_{k}\right\} \subset \mathcal{A}$ contributes a single connected arc to the boundary of $\operatorname{conv}\left(A_{i}, A_{j}, A_{k}\right)$, and we define the orientation of an ordered triple $\left(A_{i}, A_{j}, A_{k}\right)$ to be positive when the bodies appear counter-clockwise in the given order on the boundary, and to be negative otherwise. Grünbaum implicitly observes that the cyclic orderings of the triples of an orientable arrangement form an order type in his discussion on planar arrangements of simple closed curves [13, Section 3.3].

\subsection{Realizing order types}

Not every order type can be realized by a point set. In fact, most order types are not realizable, and it is NP-hard to decide if a given order type is realizable [28]. Having a notion of combinatorial type allows us to approach questions regarding realizability by bodies rather than points [15]. The smallest non-realizable order type is the Non-Pappus Configuration, a configuration of 9 elements that violates Pappus's Theorem [19, 27]. Pach and Tóth showed that the Non-Pappus Configuration can be realized by an arrangement of segments in the plane [24]. Figure 2 shows a non-realizable order type that can be realized by triangles, Goodman-Pollack's "Bad Pentagon" [8], and the authors conjecture that this order type cannot be realized by segments. In contrast to point sets, we show that any order type, and in fact any combinatorial type, can be realized by an arrangement of bodies. 

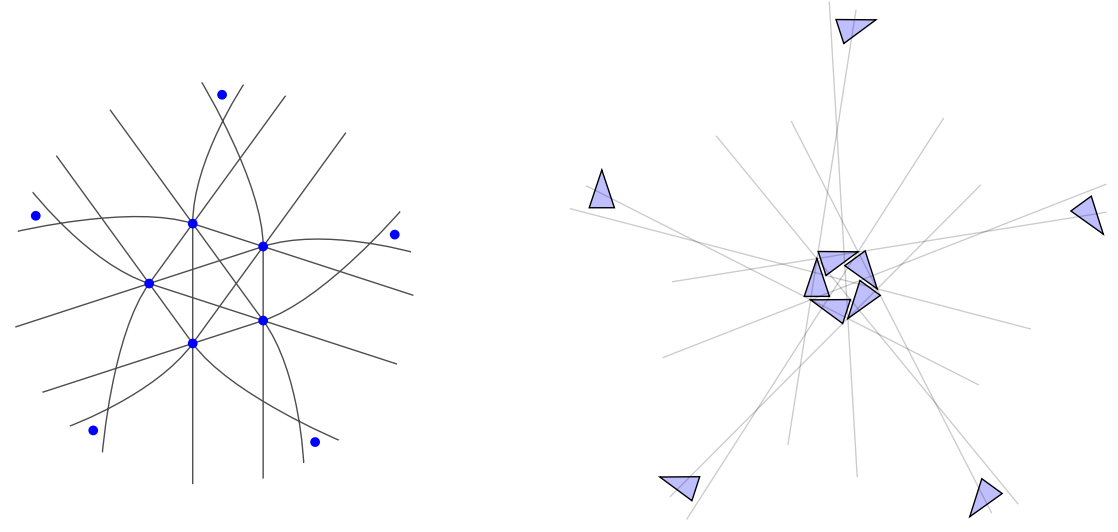

Figure 2 Two realizations of the Bad Pentagon. Left: a realization in a topological plane [8]. Right: a realization by convex sets in the Euclidean plane.

- Theorem 2. The orientations of the triples of any orientable arrangement is a simple order type. Two orientable arrangements have the same order type if and only if they have the same combinatorial type. And, every simple order type can be realized by an orientable arrangement.

We informally describe how to construct an arrangement of a given simple order type.

Proof Idea. The Folkman-Lawrence representation theorem says that any rank 3 chirotope can be realized by a (symmetric) pseudocircle arrangement; that is, by a family of simple closed curves on the sphere such that each curve is preserved by the antipodal map $(x \mapsto-x)$ and each pair of distinct curves intersect at exactly 2 points [6]. In the case of order types, there is some pair of points respectively to the left of each curve (labeled $+\infty$ ) and to the right of each curve (labeled $-\infty$ ). Pseudocircle arrangements can be swept, meaning a path from the point $-\infty$ to the point $+\infty$ can be continuously deformed while keeping its end-points fixed such that it passes through all other points on the sphere exactly once returning to its original position and it always intersects each pseudocircle at exactly one point $[9$, Theorem 2.9]. This defines a homeomorphism from the sphere with points $+\infty$ and $-\infty$ removed to the cylinder such that the image of each pseudocircle is the graph of a function $h_{i}: \mathbb{S}^{1} \rightarrow \mathbb{R}^{1}$. Furthermore, this homeomorphism can be chosen so that each function $h_{i}$ is the support function of a convex body. These convex bodies then form an orientable arrangement of the given order type.

Alternatively, such an arrangement can be constructed in the primal. A simple order type can be encoded by a sequence of permutations given by the order that a path intersects each pseudocircle as it sweeps the sphere. Consider a family of closed curves in the plane that each wind once around the origin and cross according to this sequence of permutations. By drawing these curves sufficiently close to the unit circle so that each curve is the boundary of a convex body, we obtain an orientable arrangement of the given order type.

If we bound the complexity of the bodies, then some simple order types can no longer be realized. Indeed, we show that simple order types can always be realized by $k$-gons, but may require $k$ to be arbitrarily large. 
- Theorem 3. Let $k_{n}$ be the smallest integer for which every simple order type on $n$ elements can be realized by an arrangement of $k_{n}$-gons. There are constants $c_{1}, c_{2}>0$ such that

$$
c_{1} \frac{n}{\log n} \leq k_{n} \leq c_{2} n^{2} .
$$

Proof Idea. The primal construction of Theorem 2 that realizes a simple order type by convex bodies can be done so the resulting bodies are polygons, and this gives the upper bound on $k_{n}$. We get the lower bound on $k_{n}$ by the following counting argument. Fix an integer $k$. The combinatorial type of an arrangement of $n k$-gons is determined by the order type of all $k n$ vertices of the arrangement. Therefore, the number of combinatorial types that can be realized by $n k$-gons is at most the number of order types that can be realized by $k n$ points $2^{O(k n(\log (n)+\log (k))}$, which grows more slowly than the number of simple order types on $n$ elements $2^{\Theta\left(n^{2}\right)}[10,5]$. Thus, for $n$ sufficiently large, some simple order type cannot be realized by $k$-gons, so $k \leq k_{n}$.

\subsection{Realization spaces}

An old conjecture of Ringel claimed that given two point sets with the same order type, one point set can be continuously deformed to the other while maintaining the order type [27]. This naturally leads to the study of realization spaces of order types, the set of all families of points with a fixed order type modulo projectivities. The conjecture can then be restated as, any non-empty realization space is connected. Ringel's conjecture was disproved in the early eighties, and the strongest result in this direction is Mnev's Universality Theorem [20, 21], which states that for any primary semialgebraic set $z$, there exists an order type whose realization space is homotopy equivalent to $z$. Recall that a primary semialgebraic set is the set of common solutions to some finite list of polynomial equations and strict inequalities in several real variables. This has lead to a growing body of work [1, 17, 25, 26, 29, 30].

The main objective of this paper is to extend the study of realization spaces to arrangements of bodies of a fixed combinatorial type and exhibit a trade-off between the combinatorial complexity of the bodies and the topological complexity of their realization space. The first indication of this trade-off may be observed from Theorems 2 and 3, which imply that for general convex bodies the realization space of any simple order type is non-empty, but this fails for $k$-gons. We prove two contrasting results. First, we show in Theorem 4 that Ringel's intuition is correct in this generalized context: the realization space of any combinatorial type satisfying some mild assumptions is contractible; that is, it is non-empty and has no holes of any dimension. In particular, the set of arrangements (modulo planar rotations) of convex bodies realizing any fixed simple order type is contractible, and therefore connected. Second, we show in Theorem 5 that if the bodies are restricted to polygons with at most $k$ vertices, then Mnev's Theorem generalizes. ${ }^{2}$ Specifically, we show that for every $k$ and every primary semialgebraic set $z$ there is a combinatorial type whose $k$-gon realization space is homotopy equivalent to $z$. The main ideas of the proof of Theorem 4 are given in Section 3 and the construction for Theorem 5 is given in Section 4 .

\subsection{Relationship to double pseudoline arrangements}

Pocchiola and Habert introduced an extension of chirotopes to arrangements of convex sets based on a similar notion of duality to what is presented here, called double pseudoline

2 Note that Mnev's Theorem is more specific as it deals with stable equivalence. 
arrangements [14]. The essential difference is that the dual double pseudoline of a convex set is defined as the quotient of the dual support curve by the $\mathbb{Z}_{2}$ action on the cylinder $(\theta, y) \sim(\theta,-y)$. Instead of a curve that wraps monotonically once around the cylinder, the dual double pseudoline is a curve that wraps monotonically twice around the Möbius strip. This leads to an extended notation of chirotopes that provides information about arrangements of convex sets which combinatorial types do not distinguish, such as disjointness and visibility. On the other hand, combinatorial types distinguish convex position of subarrangements and are simpler in certain respects that are crucial to the analysis in $[4,3]$ and the results of this paper.

\section{Preliminaries and main theorems}

In this section we state the main theorems and introduce definitions to be used throughout the paper. An arrangement we always mean a finite indexed non-empty collection of compact convex sets, which we call bodies.

\subsection{Genericity assumptions}

A common supporting tangent of a pair of bodies is a directed line tangent to each body such that both bodies are on its left side. In the dual, this corresponds to an intersection between two support curves. We say that a pair of bodies intersect transversally when no point of intersection is contained in a common supporting tangent. In the dual this corresponds to a pair of curves in the cylinder that cross at each point of intersection; that is, for a pair of curves that are respectively the graphs of functions $f_{1}, f_{2}$, the function $f_{1}-f_{2}$ has only isolated zeros and changes sign at each zero. An arrangement is called generic when it satisfies the following conditions:

- Each pair of bodies intersect transversally.

- No three bodies share a common supporting tangent.

- There are finitely many common supporting tangents.

A system is called generic when it satisfies the following conditions:

- Each pair of curves cross at each point of intersection.

- No three curves share a common point of intersection.

- There are finitely many crossings.

Every time we refer to an arrangement or a system, it is assumed to be generic. We will use non-generic point sets and their non-simple order types, but we do not refer to them as arrangements.

\subsection{Combinatorial type}

Let $\mathfrak{S}_{m}$ be the symmetric group on $m$ elements and $[m]=\{1, \ldots, m\}$. Given $i \in[m-1]$, the adjacent transposition $\tau_{i} \in \mathfrak{S}_{m}$ is the permutation interchanging the $i$ 'th and $i+1$ 'st entries,

$\tau_{i}\left(x_{1}, \ldots, x_{m}\right)=\left(x_{1}, \ldots, x_{i-1}, x_{i+1}, x_{i}, x_{i+2}, \ldots, x_{m}\right)$.

Let $H\left(\tau_{i}\right)=i$ denote the height of an adjacent transposition. A swap sequence $\sigma:[N] \rightarrow \mathfrak{S}_{m}$ is any sequence of adjacent transpositions such that $\sigma_{N} \circ \cdots \circ \sigma_{1}$ is the identity permutation. 


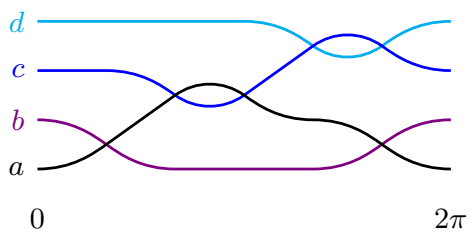

$$
\begin{aligned}
\rho & =(a, b, c, d) \\
H(\sigma) & =(1,2,2,3,1,3) \\
\overline{\rho \sigma} & =((b, a),(c, a),(a, c),(d, c),(a, b),(c, d))
\end{aligned}
$$

Figure 3 A system with its swap data $(\rho, \sigma)$ and its incidence sequence $\overline{\rho \sigma}$. Note that systems are drawn as viewed from outside the cylinder, so counter-clockwise is to the right.

Fix an index set $\mathfrak{L}$ of size $n$. A swap pair $(\rho, \sigma)$ on $\mathfrak{L}$ is a bijection $\rho:[n] \rightarrow \mathfrak{L}$ together with a swap sequence $\sigma:[N] \rightarrow \mathfrak{S}_{n}$. We define an equivalence relation ( $\stackrel{\text { swap }}{\sim}$ ) on swap pairs as follows. Let $\left(\rho^{\prime}, \sigma^{\prime}\right) \stackrel{\text { swap }}{\sim}(\rho, \sigma)$ when $\left(\rho^{\prime}, \sigma^{\prime}\right)$ can be obtained from $(\rho, \sigma)$ by performing any sequence of the following two elementary operations

- a cyclic shift

$$
\rho^{\prime}=\tau_{\sigma_{1}}(\rho), \quad \sigma_{i}^{\prime}=\sigma_{(i+1 \bmod N)}
$$

- an elementary transposition

$$
\rho^{\prime}=\rho, \quad \sigma^{\prime}=\tau_{i}(\sigma) \quad \text { provided }\left|H\left(\sigma_{i}\right)-H\left(\sigma_{i+1}\right)\right|>1 .
$$

A combinatorial type $\Omega$ on $\mathfrak{L}$ is the equivalence class $\Omega=\left\{\left(\rho^{\prime}, \sigma^{\prime}\right):\left(\rho^{\prime}, \sigma^{\prime}\right) \stackrel{\text { swap }}{\sim}(\rho, \sigma)\right\}$ of a swap pair $(\rho, \sigma)$.

To define the combinatorial type of a system $\mathcal{S}$, we order the crossings of $\mathcal{S}$ lexicographically in $\mathbb{S}^{1} \times \mathbb{R}^{1}$ where $\mathbb{S}^{1}$ is ordered according to the standard parametrization by the half-open interval $(0,2 \pi]$. Let $\rho$ be the order of the indices of each curve from bottom to top before the first crossing of the system. Let $\sigma$ be the swap sequence corresponding to each crossing. That is, let $H\left(\sigma_{i}\right)$ be 1 plus the number of curves below the $i$ 'th crossing of $\mathcal{S}$. Observe that the sequence $\sigma_{i} \circ \ldots \sigma_{1}(\rho)$ for $i=0,1, \ldots, N$ records the order of the curves in a sweep of the cylinder. The combinatorial type $\operatorname{ct}(\mathcal{S})$ of a system $\mathcal{S}$ is the equivalence class of its swap pair $(\rho, \sigma)$. The combinatorial type of an arrangement $\mathcal{A}$ is that of its dual system, and by slight abuse of notation, we write $\operatorname{ct}(\mathcal{A})=\operatorname{ct}\left(\mathcal{A}^{*}\right)$.

The incidence sequence $\overline{\rho \sigma}:[N] \rightarrow \mathfrak{L}^{2}$ of a swap pair $(\rho, \sigma)$ records the ordered pair of indices transposed by the action of each swap,

$$
{\overline{\rho \sigma_{i}}}_{i}=\left(x_{H\left(\sigma_{i}\right)+1}, x_{H\left(\sigma_{i}\right)}\right) \quad \text { where } \quad x=\sigma_{i-1} \circ \cdots \circ \sigma_{1}(\rho) .
$$

Note that the incidence sequence of equivalent swap pairs have the same multi-set of entries.

The layers of a system are the connected components of the union of curves of the system. Analogously, the layers of a combinatorial type are the connected components of the graph on $\mathfrak{L}$ defined by its incidence sequence. The depth of a combinatorial type is the number of layers excluding isolated vertices, and the depth 1 case is called non-layered.

\subsection{Realization spaces}

The full realization space $\mathcal{R}(\Omega)$ of a combinatorial type $\Omega$ is defined by

$$
\mathcal{R}(\Omega):=\left\{\mathcal{A} \in \mathcal{U}_{\mathfrak{L}}: \operatorname{ct}(\mathcal{A})=\Omega\right\}
$$

where $\mathcal{U}_{\mathfrak{L}}$ is the set of all arrangements of bodies indexed by $\mathfrak{L}$. The Hausdorff metric $d_{H}$ on compact subsets of $\mathbb{R}^{2}$ induces a metric on $\mathcal{R}(\Omega)$ by taking the maximum distance between 
bodies having the same index. That is, for $\mathcal{A}=\left\{A_{i}\right\}_{i \in \mathfrak{L}}$ and $\mathcal{B}=\left\{B_{i}\right\}_{i \in \mathfrak{L}}$,

$$
d(\mathcal{A}, \mathcal{B})=\max _{i \in \mathfrak{L}} d_{H}\left(A_{i}, B_{i}\right)
$$

- Remark. The map that takes a convex body to its support function is an isometry from the space of convex bodies with the Hausdorff metric to the space of support functions on $\mathbb{S}^{1}$ with the supremum metric.

Depending on context, it may be convenient to regard realizations of a fixed combinatorial type as "the same" when they are related by a projective transformation. Let $\mathcal{A} \stackrel{\text { proj }}{\sim} \mathcal{B}$ when they are related by an admissible projectivity; that is, an invertible projective transformation $\pi$ such that $\pi\left(A_{i}\right)=B_{i}$ for all $i \in \mathfrak{L}$ and $\pi$ is bounded and preserves orientation on the convex hull of $\bigcup \mathcal{A}$. The (projective) realization space, which we may simply call the "realization space", is the quotient space

$$
\widetilde{\mathcal{R}}(\Omega):=\mathcal{R}(\Omega) / \stackrel{\text { proj }}{\sim} .
$$

By a $k$-gon we mean a convex polygon with at most $k$ vertices. The full $k$-gon realization space is given by

$$
\mathcal{R}_{k}(\Omega):=\left\{\mathcal{A} \in \mathcal{R}(\Omega): \forall i \in \mathfrak{L} . A_{i} \text { is a } k \text {-gon }\right\}
$$

Similarly, we have the (projective) $k$-gon realization space $\widetilde{\mathcal{R}}_{k}(\Omega):=\mathcal{R}_{k}(\Omega) / \stackrel{\text { proj. }}{\sim}$ Let $\mathbb{T}^{d}=\mathbb{S}^{1} \times \cdots \times \mathbb{S}^{1}$ denote the $d$-torus, the $d$-fold product of $\mathbb{S}^{1}$.

- Theorem 4. The realization space $\widetilde{\mathcal{R}}(\Omega)$ of any non-layered combinatorial type $\Omega$ is contractible. Moreover, if $\Omega$ has depth $d>1$, then $\widetilde{\mathcal{R}}(\Omega)$ is homotopic to a $(d-1)$-torus.

- Remark. Orientable combinatorial types are always non-layered.

- Theorem 5. For every primary semialgebraic set $z$ and every positive integer $k$, there exists a combinatorial type $\Omega$ such that $\widetilde{\mathcal{R}}_{k}(\Omega)$ is homotopic to $Z$.

\section{Contractibility}

To show contractibility, we construct a standard arrangement of convex bodies for each combinatorial type by defining its dual support system. We then show that the full realization space $\mathcal{R}(\Omega)$ is equivariantly homotopic to a circle $\mathbb{S}^{1}$ by defining a deformation retraction to the subspace of rotated copies of the standard arrangement. By equivariantly homotopic we mean that the corresponding homotopy maps commute with $S O(2)$. We then pass to the (projective) realization space $\widetilde{\mathcal{R}}(\Omega)$ by identifying arrangements related by admissible projectivities. Since rotations are admissible projectivities, this defines a deformation retraction from $\widetilde{\mathcal{R}}(\Omega)$ to a point.

The deformation retraction from $\mathcal{R}(\Omega)$ to a circle will proceed in two steps. First in Lemma 6 , we deform the support system of a given arrangement to a system of the same combinatorial type that depends only on the (angular) position of each crossing; see Figure 4 Left. We can then consider just the positions of the crossings and ignore the rest of the geometry of the system. Second in Lemma 7, we move the crossings to a set of standard positions that depend only on: the given combinatorial type and the position of a certain crossing that we fix; see Figure 4 Right. The set of possible standard systems we get in the end is parametrized by the position of this fixed crossing, which defines an embedding of the circle in $\mathcal{R}(\Omega)$. The first deformation retraction depends on the following remark. 

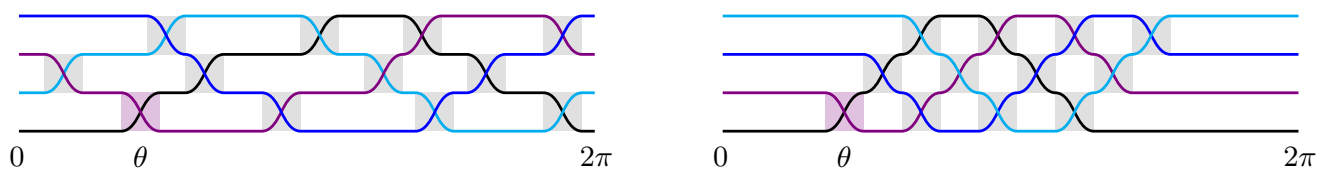

Figure 4 Left: The system $\alpha^{*}(V)$ depending on the angular positions of the crossings as given by the support configuration $V \in \mathcal{V}(\Omega)$. Right: The system $W(\theta, \delta)$ with the marked crossing fixed at angle $\theta$ obtained by rotating all other crossings of $\alpha^{*}(V)$ clockwise.

- Remark. For any pair of convex bodies $A$ and $B,(A+B)^{*}=A^{*}+B^{*}$ with Minkowski addition on the left and addition of the support functions defining the curves on the right. And, for $t \geq 0,(t A)^{*}=t\left(A^{*}\right)$. Hence, the set of all support functions is a convex cone. That is, if $g$ and $h$ are support functions, then so is $s g+t h$ for $s, t \geq 0$. Note however, that the set of dual support systems of a fixed combinatorial type is not a convex set.

\subsection{Support configurations}

The support configuration of an arrangement $\mathcal{A}$ indexed by $\mathfrak{L}$ is a labeled vector configuration $\operatorname{sc}(\mathcal{A}) \subset \mathfrak{L}^{2} \times \mathbb{S}^{1}$ which contains a triple $(i, j, \theta)$ if bodies $A_{i}, A_{j}$ have a common supporting tangent line $\ell$ that first meets $A_{i}$ and then meets $A_{j}$ and has outward normal vector $\theta$. We say labels $(i, j),\left(i^{\prime}, j^{\prime}\right)$ are disjoint when $\{i, j\} \cap\left\{i^{\prime}, j^{\prime}\right\}=\emptyset$. Note that a unit vector $\theta$ may appear in multiple elements of $\operatorname{sc}(\mathcal{A})$ with disjoint labels. Dually, $\operatorname{sc}(\mathcal{A})$ corresponds to the crossings of $\mathcal{A}^{*}$. Specifically, $(i, j, \theta) \in \operatorname{sc}(\mathcal{A})$ when curves $A_{i}^{*}$ and $A_{j}^{*}$ cross at $\theta$ with $A_{i}^{*}$ crossing downward and $A_{j}^{*}$ crossing upward. That is, the respective support functions $f_{i}, f_{j}$ of $A_{i}, A_{j}$ are equal at $\theta$ and $f_{j}-f_{i}$ is increasing at $\theta$.

Observe that the support configuration of an arrangement determines the combinatorial type of that arrangement. For a given combinatorial type $\Omega$, we will define its support configuration space $\mathcal{V}(\Omega)$, which will turn out to be the set of support configurations of all arrangements realizing $\Omega$. We first define the set of labeled configurations $\mathcal{V}(\rho, \sigma)$ corresponding to a given swap pair $(\rho, \sigma)$. Recall that $\overline{\rho \sigma}$ records the ordered pairs of indices transposed by $\sigma$ acting sequentially on $\rho$. Observe that if $(\rho, \sigma)$ is the swap pair of a system, then $\overline{\rho \sigma}_{i}$ for $i \in[N]$ is the labeling of the $i$-th crossing of the system. Recall also that we order $\mathbb{S}^{1}$ by the parametrization by $(0,2 \pi]$. Let

$$
\begin{aligned}
& \mathcal{V}(\rho, \sigma):=\left\{\left\{\left(\overline{\rho \sigma}_{i}, \theta_{i}\right): i \in[N]\right\}: \begin{array}{l}
\theta_{i} \in \mathbb{S}^{1}, \theta_{i} \leq \theta_{i+1}, \\
\theta_{i}=\theta_{i+1} \Rightarrow\left|H\left(\sigma_{i}\right)-H\left(\sigma_{i+1}\right)\right|>1
\end{array}\right\}, \\
& \mathcal{V}(\Omega):=\bigcup_{(\rho, \sigma) \in \Omega} \mathcal{V}(\rho, \sigma) .
\end{aligned}
$$

Note that a vector $\theta \in \mathbb{S}^{1}$ might appear multiple times in $\mathcal{V}(\Omega)$ with different labels provided the pairs of indices in the labels are disjoint.

We define a metric on $\mathcal{V}(\Omega)$ as follows. For a given support configuration $X$ and a given ordered pair of indices $(i, j) \in\left(\begin{array}{c}\mathfrak{L} \\ 2\end{array}\right)$, let $X_{(i, j)}:=\left\{\theta \in \mathbb{S}^{1}:(i, j, \theta) \in X\right\}$. For two support configurations, $X, Y \subset \mathfrak{L}^{2} \times \mathbb{S}^{1}$,

$$
d(X, Y)=\max _{(i, j) \in\left(\frac{\mathfrak{s}}{2}\right)} d_{H}\left(X_{(i, j)}, Y_{(i, j)}\right)
$$

where the distance between two direction vectors is given by their angle and $d_{H}$ is the corresponding Hausdorff metric on sets. 
- Lemma 6. For any combinatorial type $\Omega$, the full realization space $\mathcal{R}(\Omega)$ is non-empty and equivariantly homotopic to the support configuration space $\mathcal{V}(\Omega)$.

Proof Idea. For $\mathcal{A} \in \mathcal{R}(\Omega)$ with swap pair $(\rho, \sigma)$, we have $\operatorname{sc}(\mathcal{A}) \in \mathcal{V}(\rho, \sigma) \subset \mathcal{V}(\Omega)$, so assigning each arrangement to its support configuration defines a map sc: $\mathcal{R}(\Omega) \rightarrow \mathcal{V}(\Omega)$, which will be one direction of the homotopy equivalence.

For the other direction, we define an embedding $\alpha: \mathcal{V}(\Omega) \rightarrow \mathcal{R}(\Omega)$. For each labeled configuration $V \in \mathcal{V}(\Omega)$, we construct a system of curves $\alpha^{*}(V)=\left\{A_{i}^{*}: i \in \mathfrak{L}\right\}$ where $A_{i}^{*}=f_{i}\left(\mathbb{S}^{1}\right), f_{i}: \mathbb{S}^{1} \rightarrow \mathbb{R}^{1}$, and show that $\alpha^{*}(V)$ is the dual support system of an arrangement $\alpha(V)$ that has support configuration $V$. The system $\alpha^{*}(V)$ that we construct may be regarded as a smooth analog of Goodman's wiring diagram [7].

Fix $V \in \mathcal{V}(\Omega)$, let $V_{i} \subset \mathbb{S}^{1}$ denote the vectors of $V$ with labels involving $i$, and let $\delta$ be the minimum angular distance between any two vectors of $V$ with non-disjoint labels. For $v=(i, j, \theta) \in V$ define the open $\operatorname{arc} \Theta(v):=(\theta-\delta / 2, \theta+\delta / 2) \subset \mathbb{S}^{1}$. Now define $f_{i}$ to be constant on the complement of the $\operatorname{arcs} \Theta\left(V_{i}\right)$, and to smoothly increase or decrease by \pm 1 symmetrically about $\theta$ in each arc $\Theta(v)$ according to the label on $v \in V_{i}$; that is, $f_{i}$ increases on $\Theta(v)$ if $(j, i, \theta) \in V$ and decreases if $(i, j, \theta) \in V$ for some $j .{ }^{3}$ We can additionally require each pair $f_{i}, f_{j}$ to coincide on $V_{i} \cap V_{j}$, and this determines each subfamily of $\alpha^{*}(V)$ corresponding to a layer of $\Omega$ up to a common additive constant. A proof of this is given in the journal version. To fix this additive constant in the case of one layer, let

$$
\min _{(i, \theta) \in \mathfrak{L} \times \mathbb{S}^{1}}\left(f_{i}(\theta)+f_{i}^{\prime \prime}(\theta)\right)=1
$$

and in the case of multiple layers, let the minimum of each successively higher layer be greater than the maximum of the layer immediately below by 1 . Now the system $\alpha^{*}(V)$ defined by the functions $f_{i}$ is the dual support system of an arrangement $\alpha(V) \in \mathcal{R}(\Omega)$ that is uniquely and continuously determined by $V \in \mathcal{V}(\Omega)$, and $\operatorname{sc}(\alpha(V))=V$. This gives us a subspace $\alpha(\Omega):=\{\alpha(V): V \in \mathcal{V}(\Omega)\} \subset \mathcal{R}(\Omega)$ that is homeomorphic to $\mathcal{V}(\Omega)=\operatorname{sc}(\mathcal{R}(\Omega))$. For $\mathcal{A} \in \mathcal{R}(\Omega)$ define $\mathcal{A}_{t}:=t \alpha(\operatorname{sc}(\mathcal{A}))+(1-t) \mathcal{A}$ for $0 \leq t \leq 1$ by Minkowski addition on each body of the arrangement. Since $\operatorname{sc}(\mathcal{A})=\operatorname{sc}(\alpha(\operatorname{sc}(\mathcal{A})))$ and, as we linearly interpolate between two systems with the same crossings, the crossings remain fixed, $\operatorname{sc}\left(\mathcal{A}_{t}\right)$ is constant for all $t \in[0,1]$. Thus, $\alpha(\Omega)$ is an equivariant deformation retract of $\mathcal{R}(\Omega)$.

\subsection{Local sequences and standard configurations}

We define a deformation retraction from the support configuration space $\mathcal{V}(\Omega)$ to a subspace of standard configurations $\mathcal{W}(\Omega) \subset \mathcal{V}(\Omega)$, which is is homeomorphic to a torus. The standard configuration we choose is similar to the "compressed form" given in [18, page 31]. For this, we introduce an encoding of combinatorial type extending the local sequences of a point set. The local sequence $\lambda_{i}=\left(\lambda_{i, 1}, \ldots, \lambda_{i, n_{i}}\right)$ of $i \in \mathfrak{L}$ for a system $\mathcal{S}$ lists the indices of the curves that $S_{i}$ crosses in order according to the parametrization by $(0,2 \pi]$. Similarly, the local sequence $\lambda_{i}$ for a swap pair $(\rho, \sigma)$ is the sequence of indices $\lambda_{i, j}$ appearing together with $i$ as part of a pair $\left(\lambda_{i, j}, i\right)$ or $\left(i, \lambda_{i, j}\right)$ in the incidence sequence $\overline{\rho \sigma}$. Let $\Lambda$ denote the tableau that has $\lambda_{\rho(i)}$ as its $i$ 'th row. We say $\Lambda$ is a tableau representation of the combinatorial type $\Omega \ni(\rho, \sigma)$. We say $\Omega$ is periodic when for some $p>1$, some tableau representation $\Lambda$

3 The definition of $f_{i}$ on $\Theta(v)$ is irrelevant as long as $f_{i}$ is $C^{2}$-smooth, monotonic, symmetric about $\theta$, and varies continuously with respect to $V$. A cubic spline would suffice for this. 
is the row-wise concatenation of $p$ copies of some other tableau $\Lambda^{\prime}$. We say a pair $j, k \in \mathfrak{L}$ are adjacent in a tableau $\Lambda$ with rows $\lambda_{i}$ when

$$
\lambda_{j}=\left(k, \lambda_{j, 2}, \ldots, \lambda_{j, n_{j}}\right) \quad \text { and } \quad \lambda_{k}=\left(j, \lambda_{k, 2}, \ldots, \lambda_{k, n_{k}}\right) .
$$

- Lemma 7. For any non-layered combinatorial type $\Omega$, the support configuration space $\mathcal{V}(\Omega)$ is equivariantly homotopic to the circle $\mathbb{S}^{1}$.

Proof Idea. Assume $\Omega$ is non-periodic. The periodic case is similar, and is dealt with in the journal version. We first construct a labeled vector configuration $W(\theta, \delta)$ for $\theta \in \mathbb{S}^{1}$ and $\delta>0$ sufficiently small as follows. Let $\Lambda_{\text {min }}$ be the lexicographically minimal tableau representation of $\Omega$ for which there exists exactly one adjacent pair. Note that it is always possible to find a tableau representation of a non-layered combinatorial type with exactly one adjacent pair. We will define a sequence of configurations $W_{t}$ recursively starting from $t=0$. To start, set $\Lambda_{0}=\Lambda_{\min }, \theta_{0}=\theta, W_{0}=\emptyset$. Let $\left\{\left(i_{t, 1}, j_{t, 1}\right), \ldots,\left(i_{t, m_{t}}, j_{t, m_{t}}\right)\right\}$ be the set of all adjacent pairs in $\Lambda_{t}$ where $\left(i_{t, m}, j_{t, m}\right)$ is ordered according to the row order of $\Lambda_{t}$. Let

$$
W_{t+1}=W_{t} \cup\left\{\left(i_{t, 1}, j_{t, 1}, \theta_{t}\right), \ldots,\left(i_{t, m_{t}}, j_{t, m_{t}}, \theta_{t}\right)\right\},
$$

$\theta_{t+1}=\theta_{t}+\delta$, and let $\Lambda_{t+1}$ be the tableau obtained from $\Lambda_{t}$ by interchanging the corresponding pairs of rows and deleting the first entry from each of these rows. Eventually, $\Lambda_{T}=\emptyset$ for some minimal $T$, and we obtain a support configuration $W(\theta, \delta)$ of combinatorial type $\Omega$ where the minimum angular distance between vectors with non-disjoint labels is $\delta$. Finally, let $\mathcal{W}(\Omega)=\left\{W\left(\theta, \delta_{0}\right): \theta \in \mathbb{S}^{1}\right\}$ where $\delta_{0}=2 \pi / N$.

The unique adjacent pair of $\Lambda_{\text {min }}$ corresponds to a specific labeled vector $\tilde{v}=(i, j, \theta)$ in each configuration $V \in \mathcal{V}(\Omega)$. To define a deformation retraction sending $V$ to a configuration $W \in \mathcal{W}(\Omega)$, first fix $\tilde{v}$ and rotate each of the other vectors clockwise as much as possible without decreasing the minimum distance $\delta$ between vectors with non-disjoint labels. That is, rotated each vector $x \neq \tilde{v}$ clockwise untill the there is a vector $y$ at angular distance $\delta$ in the clockwise direction from $x$ such that $y$ has already stopped rotating and $x$ and $y$ have non-disjoint labels. Once all vectors have stopped rotating, we will have arrived at the configuration $W(\theta, \delta)$. Then, continuously rescale $\delta$ to $2 \pi / N$ keeping $\tilde{v}$ fixed. This give a deformation retraction from $\mathcal{V}(\Omega)$ to $\mathcal{W}(\Omega) \simeq \mathbb{S}^{1}$.

\subsection{Proof of contractibility}

Proof of Theorem 4. In the depth 1 case, the full realization space $\mathcal{R}(\Omega)$ is homotopic to the support configuration space $\mathcal{V}(\Omega)$ by Lemma 6 , which is homotopic to $\mathbb{S}^{1}$ by Lemma 7. Since these homotopies are equivariant and rotations are included among admissible projective transformations, the realization space $\widetilde{\mathcal{R}}(\Omega)$ is contractible.

In the depth $d>1$ case, partition $\Omega$ into layers $\Omega=\Omega_{1} \cup \cdots \cup \Omega_{d}$. If we restrict a support configuration of $\Omega$ to those vectors with labels in a layer $\Omega_{i}$, then we obtain a support configuration of $\Omega_{i}$. Hence, $\mathcal{V}(\Omega) \subset \mathcal{V}\left(\Omega_{1}\right) \times \cdots \times \mathcal{V}\left(\Omega_{d}\right)$. In the other direction, if we are given support configurations $V_{i} \in \mathcal{V}\left(\Omega_{i}\right)$, then $\bigcup_{i \in[d]} V_{i} \in \mathcal{V}(\Omega)$. Hence $\mathcal{V}(\Omega)=\mathcal{V}\left(\Omega_{1}\right) \times \cdots \times \mathcal{V}\left(\Omega_{d}\right)$, and therefore by Lemmas 6 and $7, \mathcal{R}(\Omega)$ is homotopic to $\mathbb{T}^{d}$, so the realization space $\widetilde{\mathcal{R}}(\Omega)$ is homotopic to a $(d-1)$-torus $\mathbb{T}^{d-1}$.

\section{$4 \quad$ Universality}

We prove the following slightly more specific result. 
- Lemma 8. For any $k$ order types $\chi_{1}, \ldots, \chi_{k}$ on $[n]$, where at least two are distinct, there is a combinatorial type $\Omega$ on $[n]$ such that its $k$-gon realization space $\widetilde{\mathcal{R}}_{k}(\Omega)$ is homotopy equivalent to $\widetilde{\mathcal{R}}_{1}\left(\chi_{1}\right) \times \cdots \times \widetilde{\mathcal{R}}_{1}\left(\chi_{k}\right)$.

Proof of Theorem 5. Fix a primary semialgebraic set $z$ and $k>1$. Let $\chi_{1}$ be the order type of the Mnev point set with point realization space homotopic to $z$. Let $\chi_{2}, \ldots, \chi_{k}$ all be the order type of $n$ points in convex position. Note that the point realization space of $n$ points in convex position is contractible. With this, the $k$-gon realization space of $\Omega$ from Lemma 8 is also homotopic to $z$.

To show Lemma 8 , we construct a combinatorial type $\Omega$ such that for every realization $\mathcal{A}$ of $\Omega$ by $k$-gons, the vertices of each $k$-gon can be labeled. That is, each vertex can be uniquely identified using only information encoded in the combinatorial type. Note that this is not possible in general, as combinatorial type does not provide information about individual vertices directly. Furthermore, we construct $\Omega$ so that the order type of the vertices of $\mathcal{A}$ is the same in every realization and each $\chi_{i}$ appears as a subset of the vertices.

We define $\Omega$ in two ways: in the primal we construct an arrangement of $k$-gons, then in the dual we construct a system of curves. First index the order types $\chi_{i}$ so that the cyclic ordering $\chi_{1}, \chi_{2}, \ldots, \chi_{k}, \chi_{1}, \ldots$ is not periodic with period smaller than $k$. This is possible by the assumption that there are at least two distinct order types.

\subsection{The primal construction}

The primal construction $\mathcal{A}=\left\{A^{1}, \ldots, A^{n}\right\}$ depends on certain arbitrary choices that will not affect the combinatorial type. Assume for the primal construction that each $\chi_{i}$ is realizable; the non-realizable case is defined by the dual construction only.

Let $\mathcal{A}_{0}$ be an arrangement of $2 k$ points in convex position denoted by $a_{1}^{1}, a_{1}^{n}, a_{2}^{1}, a_{2}^{n}, \ldots$, $a_{k}^{1}, a_{k}^{n}$ in counter-clockwise order, such that the lines $\ell_{i}$ spanning $a_{i}^{n}$ and $a_{i+1}^{1}$ bound a convex $k$-gon $B .{ }^{4}$ Observe that $B \backslash \operatorname{conv}\left(\mathcal{A}_{0}\right)$ consists of $k$ triangular regions. We construct $\mathcal{A}$ by placing a point set realizing one of the $\chi_{i}$ in each of these traingular regions, then we define the $k$-gons $A^{s}$ to have vertices consisting of one point from each region; see Figure 5 for an example with $n=6, k=4$.

Let $\chi_{i}$ be defined on the index set $\left\{\left(\begin{array}{l}1 \\ i\end{array}\right), \ldots,\left(\begin{array}{l}n \\ i\end{array}\right)\right\}$, and let $\mathcal{P}_{i}=\left\{p_{i}^{1}, \ldots, p_{i}^{n}\right\}$ be a realization of $\chi_{i}$. Furthermore, let $\chi_{i}$ indexed so that $p_{i}^{1}$ and $p_{i}^{2}$ appear on the boundary of the convex hull of $\mathcal{P}_{i}$ and the local sequence of $p_{i}^{1}$ is $p_{i}^{2}, p_{i}^{3}, \ldots, p_{i}^{n}$. That is, the angles $\theta_{i}^{s}$ at $p_{i}^{1}$ from the semiline through $p_{i}^{2}$ to the semiline through $p_{i}^{s}$ are increasing in the counter-clockwise direction, $0=\theta_{i}^{2}<\theta_{i}^{3}<\cdots<\theta_{i}^{n}<\pi$. Note that this implies $p_{i}^{n}$ is also on the boundary of the convex hull of $\mathcal{P}_{i}$, which we will call the convex boundary for short. Now augment $\mathcal{P}_{i}$ by two points as follows. Let $\mathcal{Q}_{i}=\mathcal{P}_{i} \cup\left\{q_{i}^{1}, q_{i}^{n}\right\}$ such that $p_{i}^{n}, q_{i}^{1}, q_{i}^{n}, p_{i}^{1}$ appear consecutively in counter-clockwise order on the convex boundary of $Q_{i}$ and no line through any two points of $\mathcal{P}_{i}$ separates the points $q_{i}^{1}, q_{i}^{n}, p_{i}^{1}$. Note that this uniquely determines the order type of $Q_{i}$; see Figure 5 (left). Now define projective transformations $\phi_{i}$ such that $\phi_{i}\left(q_{i}^{n}\right)=a_{i-1}^{n}, \phi_{i}\left(p_{i}^{1}\right)=a_{i}^{1}$, $\phi_{i}\left(p_{i}^{n}\right)=a_{i}^{n}, \phi_{i}\left(q_{i}^{1}\right)=a_{i+1}^{1}$, and let $\mathcal{P}=\left\{a_{1}^{1}, a_{1}^{2}, \ldots, a_{2}^{1}, \ldots, a_{n}^{n}\right\}$ where $a_{i}^{s}=\phi_{i}\left(p_{i}^{s}\right)$. Finally, let $\mathcal{A}=\left\{A^{1}, \ldots, A^{n}\right\}$ where $A^{s}=\operatorname{conv}\left(\left\{a_{1}^{s}, a_{2}^{s}, \ldots, a_{k}^{s}\right\}\right)$, and let $\Omega$ denote the combinatorial type of $\mathcal{A}$.

${ }^{4}$ Here subscripts are indices over $\mathbb{Z}_{k}$, so in particular $\ell_{k}$ is the line spanning $a_{k}^{n}$ and $a_{1}^{1}$. 


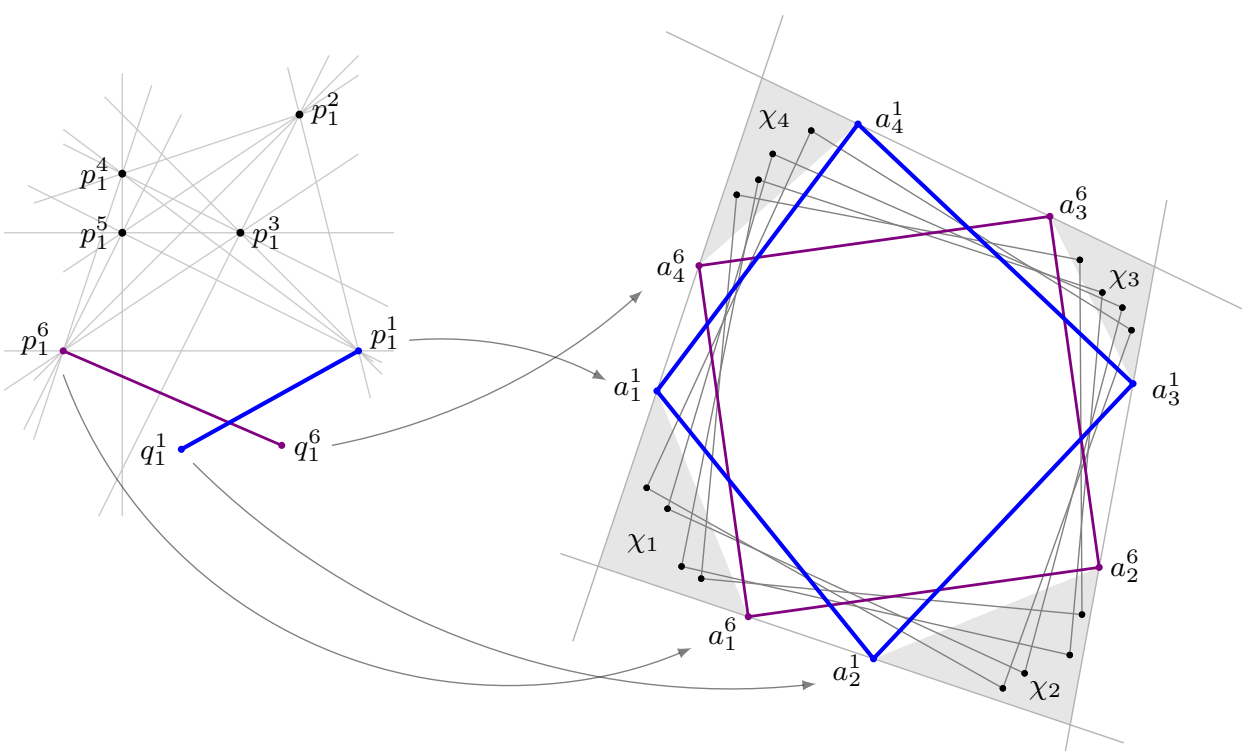

Figure 5 The point set $\mathcal{P}_{1}$ on the left is mapped to points on the right by the projective transformation determined by $p_{1}^{1} \mapsto a_{1}^{1}, q_{1}^{1} \mapsto a_{2}^{1}, p_{1}^{6} \mapsto a_{1}^{6}, q_{1}^{6} \mapsto a_{4}^{6}$

\subsection{Path systems}

We call the graph of some indexed family of functions defined over an interval a path system. We say two path systems are equivalent when the are related by an orientation preserving self-homeomorphism of the plane that also preserves indices and the orientations of the paths. We will always assume that the end-points of a path system are all distinct, and that the paths satisfy the same genericity conditions given in Subsection 2.1 for systems of curves. For path systems $\mathcal{L}_{1}, \mathcal{L}_{2}$ over intervals $I_{1}, I_{2} \subset \mathbb{R}$ with the same number of paths, the concatenation $\mathcal{L}_{1} \cdot \mathcal{L}_{2}$ is the path system obtained by identifying the right edge of $I_{1} \times \mathbb{R}$ with the left edge of $I_{2} \times \mathbb{R}$ by a homeomorphism sending the right end-points of $\mathcal{L}_{1}$ to the left end-points of $\mathcal{L}_{2}$. Here indices must be dealt with appropriately. If the left end-points of $\mathcal{L}_{1}$ appear in the same order as the right end-points, then we may form a system of curves $\circlearrowleft \mathcal{L}_{1}$ by identifying the left and right edges of $I_{1} \times \mathbb{R}$ by a homeomorphism that identifies the left and right end-points of each path in $\mathcal{L}_{1}$. Let $\uparrow \mathcal{L}_{1}$ denote the path system obtained by flipping $\mathcal{L}_{1}$ vertically by the map $(x, y) \mapsto(x,-y)$. Given an order type $\chi$, we say a path

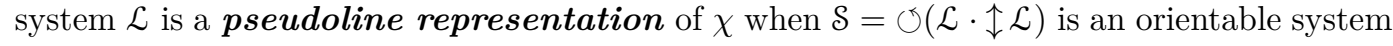
with order type $\chi$ as in Theorem 2 . We say an element $i$ is on the convex boundary of $\chi$ when the corresponding curve $S_{i}$ appears on the upper envelope of a corresponding system $\mathcal{S}$.

- Remark. For each element $i$ on the convex boundary of an order type $\chi$, there is a unique class of equivalent pseudoline representations $\mathcal{L}$ where $L_{i}$ starts as the top most path and crosses all other paths, thereby going to the bottom, before any other crossings occur.

\subsection{The dual construction}

Let $\chi_{i}$ be an order type on elements $\left\{\left(\begin{array}{l}1 \\ i\end{array}\right), \ldots,\left(\begin{array}{l}n \\ i\end{array}\right)\right\}$ indexed as in the primal construction, and let $\mathcal{L}_{i}$ be a pseudoline representation of $\chi_{i}$ with paths $L_{i}^{1}, \ldots, L_{i}^{n}$ such that $L_{i}^{1}$ starts at the top and crosses all other paths first as in the above remark. Let $\mathcal{C}=\left\{C_{1}, \ldots, C_{k}\right\}$ be the dual system of $k$ points in convex position indexed in counter-clockwise order, and observe that each curve $C_{i}$ appears exactly once on the upper envelope and once on the 


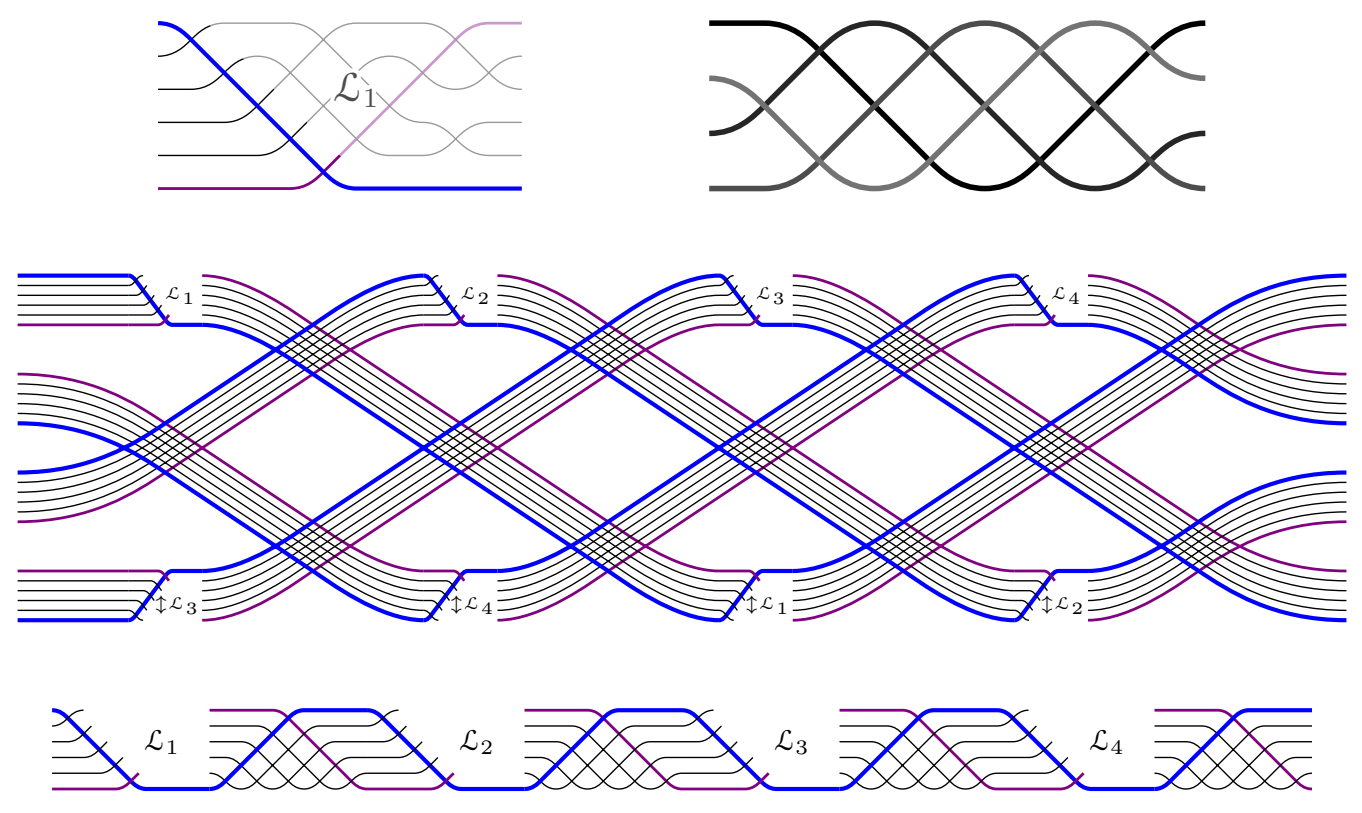

Figure 6 Top left: The pseudoline representation $\mathcal{L}_{1}$ of $\chi_{1}$. Top right: The system $\mathcal{C}$. Center: The system $\mathcal{S}$. Bottom: The system $\mathcal{T}$ of combinatorial type $\Omega$.

lower envelope of $\mathcal{C}$. Let $\mathcal{S}$ be a system of curves where each curve $C_{i} \in \mathcal{C}$ is replaced by $n$ curves $\left\{S_{i}^{1}, \ldots, S_{i}^{n}\right\}$ in a small tubular neighborhood about $C_{i}$ crossing to form a copy of $\mathcal{L}_{i}$ above all other curves of $\mathcal{S}$ and a copy of $\left\{\mathcal{L}_{i}\right.$ below all other curves of $\mathcal{S}$. Let $T^{s}$ be the upper envelope of the curves $S_{1}^{s}, \ldots, S_{k}^{s}$, and let $\mathcal{T}=\left\{T^{1}, \ldots, T^{n}\right\}$. Equivalently, let $\mathcal{U}$ be the path system of size $n$ where each path from bottom to top crosses all paths below itself (beginning with the bottom path crossing no other paths and ending with the top path crossing all other paths), and let $\mathcal{T}=\circlearrowleft\left(\mathcal{L}_{1} \cdot \mathcal{U} \cdot \mathcal{L}_{2} \cdot \mathcal{U} \cdots \mathcal{L}_{k} \cdot \mathcal{U}\right)$. Finally, define $\Omega$ to be the combinatorial type of $\mathcal{T}$. See Figure 6 for an example with $n=6, k=4$.

Proof Idea of Lemma 8. Each body $A^{t}$ for $t \in\{2, \ldots, n\}$ appears $k$ times on the convex boundary of $\left\{A^{1}, A^{t}\right\}$, so $A^{1}$ and $A^{t}$ must each have exactly $k$ vertices. In this way, the vertices of $\mathcal{A}$ can be partitioned into $k$ parts consisting of one vertex from each $A^{s}$ for $s \in[n]$, and these parts realize each $\chi_{i}$ in the given cyclic order. Since the sequence of $\chi_{i}$ is not periodic with period smaller than $k$, each part is associated to each $\chi_{i}$ in a unique way. This defines a map $\varphi: \widetilde{\mathcal{R}}_{k}(\Omega) \rightarrow \widetilde{\mathcal{R}}_{1}\left(\chi_{i}\right)$ such that $\varphi_{1} \times \cdots \times \varphi_{k}: \widetilde{\mathcal{R}}_{k}(\Omega) \rightarrow \widetilde{\mathcal{R}}_{1}\left(\chi_{1}\right) \times \cdots \times \widetilde{\mathcal{R}}_{1}\left(\chi_{k}\right)$ is a fiber bundle with contractible fiber, so $\widetilde{\mathcal{R}}_{k}(\Omega)$ is homotopic to $\widetilde{\mathcal{R}}_{1}\left(\chi_{1}\right) \times \cdots \times \widetilde{\mathcal{R}}_{1}\left(\chi_{k}\right)$.

\section{References}

1 Anders Björner, Michel Las Vergnas, Bernd Sturmfels, Neil White, and Günter M. Ziegler. Oriented matroids, volume 46 of Encyclopedia of Mathematics and its Applications. Cambridge University Press, 1999.

2 Raghavan Dhandapani, Jacob E. Goodman, Andreas Holmsen, and Richard Pollack. Interval sequences and the combinatorial encoding of planar families of pairwise disjoint convex sets. Rev. Roum. Math. Pures Appl, 50(5-6):537-553, 2005.

3 Michael Gene Dobbins, Andreas Holmsen, and Alfredo Hubard. Regular systems of paths and families of convex sets in convex position. To appear in Transactions of the AMS. 
4 Michael Gene Dobbins, Andreas Holmsen, and Alfredo Hubard. The Erdős-Szekeres problem for non-crossing convex sets. Mathematika, 60(2):463-484, 2014.

5 Stefan Felsner and Pavel Valtr. Coding and counting arrangements of pseudolines. Discrete E) Computational Geometry, 46(3):405-416, 2011.

6 Jon Folkman and Jim Lawrence. Oriented matroids. Journal of Combinatorial Theory, Series B, 25(2):199-236, 1978.

7 Jacob E. Goodman. Proof of a conjecture of Burr, Grünbaum, and Sloane. Discrete Mathematics, 32(1):27-35, 1980.

8 Jacob E. Goodman and Richard Pollack. On the combinatorial classification of nondegenerate configurations in the plane. Journal of Combinatorial Theory, Series A, 29(2):220-235, 1980.

9 Jacob E. Goodman and Richard Pollack. Semispaces of configurations, cell complexes of arrangements. Journal of Combinatorial Theory, Series A, 37(3):257-293, 1984.

10 Jacob E. Goodman and Richard Pollack. Upper bounds for configurations and polytopes in $\mathbb{R}^{d}$. Discrete \& Computational Geometry, 1(1):219-227, 1986.

11 Jacob E. Goodman and Richard Pollack. The combinatorial encoding of disjoint convex sets in the plane. Combinatorica, 28(1):69-81, 2008.

12 Helmut Groemer. Geometric applications of Fourier series and spherical harmonics, volume 61 of Encyclopedia of Mathematics and its Applications. Cambridge University Press, 1996.

13 Branko Grünbaum. Arrangements and spreads. American Mathematical Society, 1972.

14 Luc Habert and Michel Pocchiola. Arrangements of double pseudolines. In Proceedings of the 25th Annual Symposium on Computational Geometry, pages 314-323. ACM, 2009.

15 Alfredo Hubard. Erdős-Szekeres para convexos. Bachelor's thesis, UNAM, 2005.

16 Alfredo Hubard, Luis Montejano, Emiliano Mora, and Andrew Suk. Order types of convex bodies. Order, 28(1):121-130, 2011.

17 Michael Kapovich and John J. Millson. Universality theorems for configuration spaces of planar linkages. Topology, 41(6):1051-1107, 2002.

18 Donald E. Knuth. Axioms and hulls, volume 606 of Lecture Notes in Computer Science. Springer-Verlag, 1992.

19 Friedrich Levi. Die teilung der projektiven ebene durch gerade oder pseudogerade. Ber. Math.-Phys. Kl. Sächs. Akad. Wiss, 78:256-267, 1926.

20 Nicolai E. Mnev. Varieties of combinatorial types of projective configurations and convex polytopes. Doklady Akademii Nauk SSSR, 283(6):1312-1314, 1985.

21 Nicolai E. Mnev. The universality theorems on the classification problem of configuration varieties and convex polytopes varieties. In Topology and Geometry: Rohlin seminar, pages 527-543. Springer, 1988.

22 Mordechai Novick. Allowable interval sequences and line transversals in the plane. Discrete 86 Computational Geometry, 48(4):1058-1073, 2012.

23 Mordechai Novick. Allowable interval sequences and separating convex sets in the plane. Discrete $\&$ Computational Geometry, 47(2):378-392, 2012.

24 János Pach and Géza Tóth. Families of convex sets not representable by points. In Algorithms, architectures and information systems security, volume 3, page 43. World Scientific, 2008.

25 Arnau Padrol and Louis Theran. Delaunay triangulations with disconnected realization spaces. In Proceedings of the 30th Annual Symposium on Computational Geometry, pages 163-170. ACM, 2014.

26 Jürgen Richter-Gebert. Realization spaces of polytopes, volume 1643 of Lecture Notes in Mathematics. Springer Verlag, 1996. 
27 Gerhard Ringel. Teilungen der ebene durch geraden oder topologische geraden. Mathematische Zeitschrift, 64(1):79-102, 1956.

28 Peter W. Shor. Stretchability of pseudolines is NP-hard. In Applied Geometry and Discrete Mathematics: The Victor Klee Festschrift, volume 4, pages 531-554. American Mathematical Society, 1991.

29 Yasuyuki Tsukamoto. New examples of oriented matroids with disconnected realization spaces. Discrete \& Computational Geometry, 49(2):287-295, 2013.

30 Ravi Vakil. Murphy's law in algebraic geometry: badly-behaved deformation spaces. Inventiones Mathematicae, 164(3):569-590, 2006. 